\title{
Workflow of Cryo-Electron Microscopy and Status of Domestic Infrastructure
}

\author{
Ki Ju Choi, Jae In Shin, Sung Hun Lee* \\ Division of Materials and Structural Analysis, Thermo Fisher Scientific Inc., Suwon 16229, Korea
}

*Correspondence to:

Lee $\mathrm{SH}_{\text {, }}$

(iD) http://orcid.org/0000-0002-4361-9116

Tel: +82-31-888-9900

Fax: +82-31-888-9901

E-mail: SungHun.Lee@fei.com

Received March 2, 2018

Revised March 26, 2018

Accepted March 26, 2018
Cryo-electron microscopy (cryo-EM) allows the analysis of the near-native structures of samples such as proteins, viruses, and sub-cellular organelles at the sub-nano scale. With the recent development of analytical methods, this technique has achieved remarkable results. The importance of cryo-EM gained wide recognition due to last year's award of the Nobel Prize in Chemistry. To help promote the knowledge of this technique, this paper introduces the basic workflows of cryo-EM and domestic cryo-EM service institutes.

Key Words: Cryo-electron microscopy, Workflow, Single particle analysis, Cryo-electron tomography

\section{INTRODUCTION}

Cryo-electron microscopy (cryo-EM) is an electron microscopy (EM) technique that can analyze the nearnative structure of radiation sensitive samples in cryogenic conditions. This analytical technique appeared toward the end of the 1980s and is based on the phenomenon of the transition of water to amorphous vitreous ice when the water is cooled rapidly (Dubochet et al., 1988). The ice renders the state of a sample existing in a liquid environment, such as a purified protein in a buffer solution, observable. Such freezing is free from problems such as the shrinkage of a sample due to drying that occurs in conventional sample preparation and the reduction in contrast resolution caused by heavy metal adsorption. Ultimately, cryo-EM allows for the analysis at nano or sub-nano scales of the near-native structure of materials, such as liposomes, as well as biological specimens, such as protein particles and viruses in liquids. Cryo-EM techniques have recently been attracting attention because they can effectively overcome the limitations of X-ray crystallography (XRC) or nuclear magnetic resonance (NMR) spectroscopy. In conventional XRC analysis, the preparation of protein crystals is difficult, as is the analysis of protein samples. In NMR spectroscopy analysis, protein samples with overly large or complex structures are difficult to analyze. In addition to the high performance of cryo-EM equipment, the development of rapid freezing methods and the development of three-dimensional (3D) reconstruction image processing techniques from two-dimensional (2D) images have played important roles in reconstructing the structures of these complex proteins at the molecular level. Recently, a large number of protein structures have been registered on sites such as Protein Data Bank (PDB) and Electron Microscopy Data Bank (EMDB).

\section{SYSTEM ENVIRONMENT OF TOOLS FOR CRYO-EM ANALYSIS}

Although not a prerequisite for cryo-EM analysis, several requirements must be met to achieve good results. Biological specimens that have not been treated in cryogenic conditions must be analyzed.

(1) To maintain the stable temperature of a specimen, the drift (Fig. 1B) from the temperature change through a shielding device (Fig. 1A) that surrounds the specimen in the transmission electron microscope (TEM) column at low temperature must be stabilized.

(2) A low-dose acquisition system (Fig. 1C) is needed to distribute the focusing area around a specimen to minimize specimen deformation caused by electron-beam exposure

(a) This is an open-access article distributed under the terms of the Creative Commons Attribution Non-Commercial License (http://creativecommons.org/licenses/by-nc/4.0) which permits unrestricted noncommercial use, distribution, and reproduction in any medium, provided the original work is properly cited.

Copyrights @ 2018 by Korean Society of Microscopy 

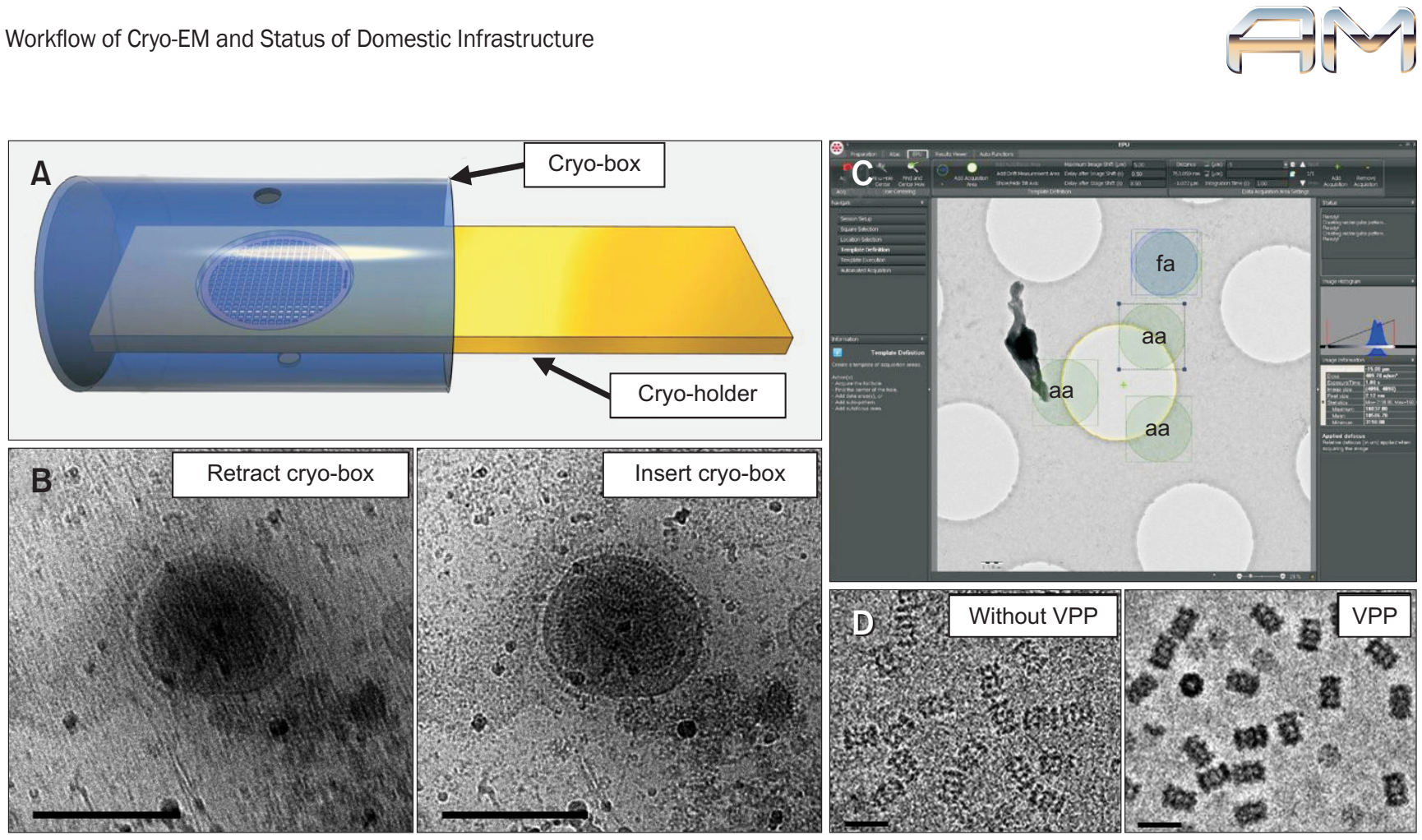

Fig. 1. Required conditions for tools used in cryo-electron microscopy. Vitrified ice samples respond sensitively to ambient temperature changes. Therefore, a protective shield, such as a cryo-box or cryo-shield, is essential for keeping the temperature stable (A). (B) The cryo-box minimizes the drift of samples caused by temperature variations. (C) The low-dose system is used to minimize exposure to the electron beam during the acquisition. The positions of the focus area (fa) and the acquired area (aa) are set differently. (D) Volta phase plate (VPP) provides a high-contrast image with minimal defocus values. Eventually, the presence of these conditions helps to achieve high-resolution results. Scale bars=200 $\mathrm{nm}$ (B) and $20 \mathrm{~nm}$ (D). Fig. D is adapted from the article of Danev and Baumeister (2016) (eLife 5, 13046) in accordance with the Creative Commons Attribution 4.0 International (CC BY 4.0) license.

(Grassucci et al., 2008).

(3) A phase plate (Fig. 1D; Danev \& Baumeister, 2016) can be used to increase the contrast of an image without reducing the resolution with minimal defocus value during image acquisition and complementary metal-oxide semiconductor (CMOS)-based detectors with high sensitivity (detective quantum efficiency) reduce radiation beam damage of samples under low electron dose conditions (McMullan et al., 2014), and at the same time, through data acquisition with more than 30 frames per second and the function of drift correction, it enables us to obtain high-resolution images (Bai et al., 2013; Bammes et al., 2012; Li et al., 2013).

(4) In addition to hardware-based systems, the role of software systems to acquire and process large amounts of image data automatically is also important for analyzing many protein structures and intracellular 3D structures. High-quality image data yield high-resolution results, and an automated image acquisition system provides researchers with an efficient analytical environment.

\section{WORKFLOW OF CRYO-SINGLE PARTICLE ANALYSIS}

The single-particle analysis (SPA) technique has been used for a long time via the negative staining method, but recently developed cryo-EM analytical techniques have become the main techniques for analyzing the high-resolution structures of proteins. Under homogenous, high concentration and high purity conditions, SPA is performed by immobilizing a sample of protein particles in the form of a thin film of vitrified ice using a refrigerant (liquid ethane) of $-170^{\circ} \mathrm{C}$ (Fig. 2 [Carroni \& Saibil, 2016]; left). Then, low-dose electron beams at low temperatures are passed through the sample to obtain images without causing radiation damage (Fig. 2; center) (Frank et al., 1981). Software such as EMAN and Relion, uses techniques such as particle picking, particle alignment, 2D averaging, defocus determination, contrast transfer function (CTF) correction, particle alignment and classification with the initial model, and final structure mapping (Fig. 2; right) to create high-resolution 3D images of protein particles at the molecular level (Scheres, 2012).

\section{WORKFLOW OF CRYO-ELECTRON TOMOGRAPHY (CRYO-ET)}

Electron tomography (ET) analysis has been a long-established analytical method, but recent advances in cryo-TEM analysis have made the latter a new trend. Cryo-TEM based 


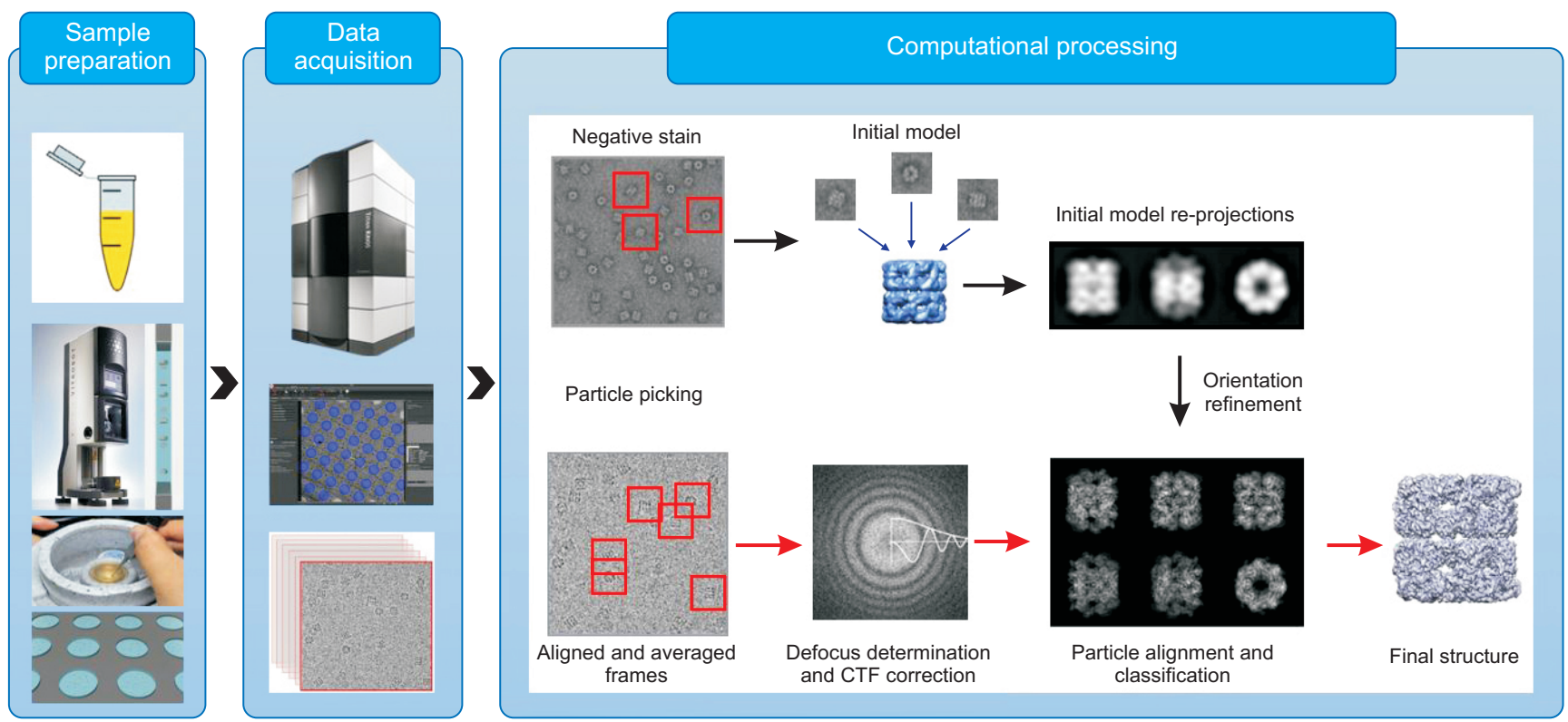

Fig. 2. Workflow of single-particle analysis for protein reconstruction. First of all, purified samples should be screened through negative staining to confirm adequate concentration and homogeneity. Classification and averaging can create better contrasting images. Used to construct initial models for orientation refinement, which is done by class averaging. In sequence, vitrified specimens of proteins are prepared by plunge freezing, after which three-dimensional images are created by computational processes such as particle two-dimensional classification, averaging from aligned and driftcorrected subframe images, defocus determination and contrast transfer function (CTF) correction, and orientation refinement with initial models to determine resolution. Adapted and modified from Carroni and Saibil (2016) (Methods 95, 78-85) in accordance with the Creative Commons Attribution 4.0 International (CC BY 4.0) license.

tomography analysis can provide higher resolution images of the 3D structural analysis of the near-native environment of a sample (Briggs, 2013) and has the advantage of in situ analysis, such as multi-scale particles and membrane proteins, which are difficult to analyze by SPA (Schur et al., 2013). Cryo-ET analysis can be approached in three main ways.

\section{Whole Cell Tomography: Plunge Freezing (Proteins, Viruses, and Cells), Cryo-TEM Tomography}

This is the method for obtaining tomographic data with preparation done by the plunge freezing method, in which the particles, such as viruses, liposomes, and cells, have been cultured on a supported film grid and rapidly frozen in liquid ethane (Fig. 3; plunge freezing). We can obtain the data through a relatively simple preprocessing, but in the case of a cell, thick portions, such as the nuclear perimeter, have very limited observable regions because an electron beam cannot transmit through such thick barriers. The samples that can be mainly analyzed in this way are viruses, bacteria, single cells (edge areas through which the electron beam can penetrate), liposomes, exosomes, etc.

\section{Cryo-EM of Vitreous Ice Section (CEMOVIS): Plunge Freezing or High-Pressure Freezing (HPF) (Cell and} Tissue), Cryo-Ultramicrotome, Cryo-TEM Tomography

This is a method for obtaining tomographic data (Fig. 3;
CEMOVIS) with a cryo-ultramicrotome at 100 to $300 \mathrm{~nm}$ thickness and maintaining the frozen state after rapidly freezing large samples using plunge or high-pressure freezing. This technique allows for the analysis of the microstructure inside the samples irrespective of their thickness, but preparing the section using the cryo-ultramicrotome is difficult, as is obtaining the sections of desired regions. In addition, artifacts may be formed due to breakages of the frozen sections or knife markers during cryosectioning.

\section{Cryo-FIB Milled Lamellae: Plunge Freezing or HPF (Cell and Tissue), Cryo-FIB Milling, Cryo-TEM Tomography}

Focused ion beams (FIB), which are mainly used for thin film fabrications of semiconductors or material samples, are also used in the processing of biological samples for ET. A cryofixed sample is milled using an ion-beam around a precise target position in an FIB chamber equipped with the cryostage (Fig. 3; cryo-FIB milling) and the tomographic data are obtained by cryo-TEM, which causes less contamination than does cryosectioning and allows the samples to be prepared in a relatively short time, making efficient tomographic analysis possible. However, few institutes have FIB equipment with cryo-stage and lack of accessibility is often the case in the domestic situation, where experience in the preparation of biological samples is scarce. 

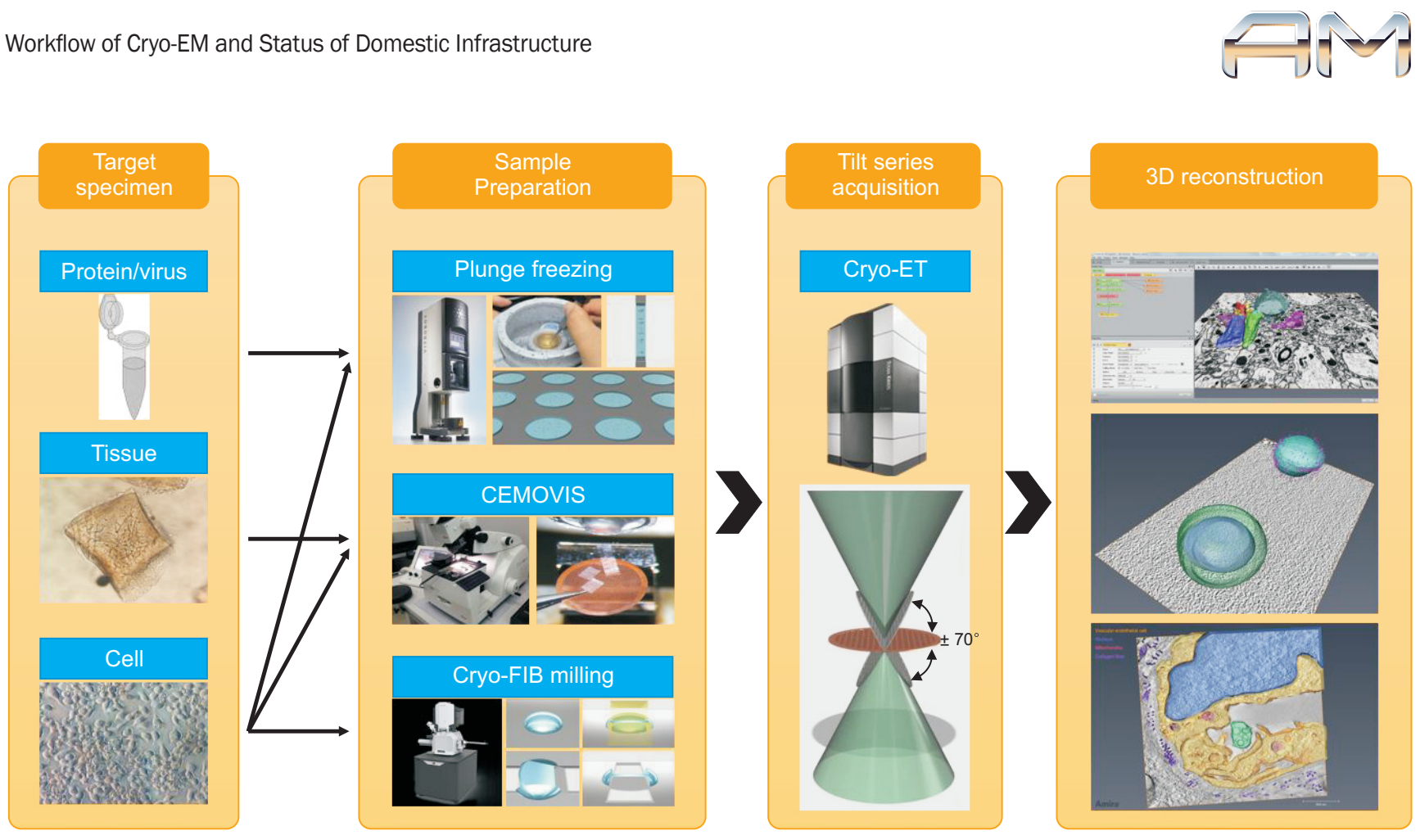

Fig. 3. Schematic of cryo-electron tomography (cryo-ET) workflow. Cryo-ET analysis can be approached in three main ways by whole cell tomography (plunge freezing), cryo-electron microscopy of vitreous ice sections (CEMOVIS), and cryo-focused ion beams (FIB) milled lamellae.

Table 1. Domestic infrastructure of cryo-electron microscopy for life sciences

\begin{tabular}{|c|c|c|c|c|c|c|c|c|c|}
\hline \multirow{2}{*}{ Organization } & \multirow{2}{*}{ Region } & \multirow{2}{*}{ TEM model (kV) } & \multicolumn{2}{|c|}{ Preparation } & \multicolumn{3}{|c|}{$\begin{array}{c}\text { Application } \\
\text { (cryo-TEM base) }\end{array}$} & \multicolumn{2}{|c|}{$\begin{array}{c}\text { Access } \\
\text { user }\end{array}$} \\
\hline & & & $\begin{array}{l}\text { Plunge } \\
\text { freezing }\end{array}$ & $\begin{array}{l}\text { Cryo- } \\
\text { section }\end{array}$ & $\begin{array}{l}\text { SPA or } \\
\text { Screening }\end{array}$ & Tomography & 2D imaging & Internal & External \\
\hline KIST & Seoul & FEI Tecnai F20 (200) & 0 & 0 & 0 & 0 & 0 & 0 & 0 \\
\hline NICEM & Seoul & FEI Talos L120C (120) & $\bigcirc$ & & O & O & $\bigcirc$ & 0 & $\bigcirc$ \\
\hline \multirow[t]{4}{*}{ KBSI } & Ochang & FEI Titan Krios (300) & 0 & 0 & 0 & 0 & 0 & 0 & 0 \\
\hline & & FEI Tecnai $12(120)$ & 0 & 0 & 0 & 0 & 0 & 0 & 0 \\
\hline & & JEOL JEM 1400 (120) & 0 & 0 & 0 & & 0 & 0 & 0 \\
\hline & Chuncheon & JEOL JEM 2100F (200) & $\bigcirc$ & & 0 & & $\bigcirc$ & 0 & 0 \\
\hline KCDC & Osong & ZEISS Libra 120 (120) & 0 & 0 & 0 & & 0 & 0 & \\
\hline KAIST & Daejeon & FEI Tecnai 12 (120) & 0 & & 0 & & 0 & 0 & \\
\hline DGIST & Daegu & FEI Tecnai F20 (200) & 0 & 0 & 0 & & 0 & 0 & 0 \\
\hline KNU & Daegu & HITACHI HT7700 (120) & & 0 & & 0 & 0 & 0 & 0 \\
\hline UNIST & Ulsan & JEOL JEM 1400 (120) & $\bigcirc$ & & 0 & & 0 & 0 & 0 \\
\hline
\end{tabular}

KIST, Korea Institute of Science and Technology; NICEM, National Instrumentation Center for Environmental Management; KBSI, Korea Basic Science Institute; KCDC, Korea Centers for Disease Control and Prevention; KAIST, Korea Advanced Institute of Science and Technology; DGIST, Daegu Gyeongbuk Institute of Science and Technology; KNU, Kyungpook National University; UNIST, Ulsan National Institute of Science and Technology; TEM, transmission electron microscope; SPA, single-particle analysis; 2D, two-dimensional.

\section{DOMESTIC INFRASTRUCTURE AND NECESSITY FOR CRYO-TEM ANALYSIS}

As we have seen, cryo-EM analysis provides a new basis for understanding and approaching in structural research in medical biology, and may become widely to study disease. Thus, the extent to which the domestic cryoEM infrastructure should be developed and what such development requires is worth considering. Table 1 lists the very few cryo-TEM analytical service institutes available in Korea. To build an advanced EM infrastructure, nurturing skilled technicians and investments in equipment at the national level are necessary. In addition, cryo-EM analysis must be shared by many researchers. 


\section{CONFLICT OF INTEREST}

No potential conflict of interest relevant to this article was reported.

\section{REFERENCES}

Bai X C, Fernandez I S, McMullan G, and Scheres S H W (2013) Ribosome structures to near atomic resolution from thirty thousand cryo-EM particles. eLIFE 2, 461.

Bammes B E, Rochat R H, Jakana J, Chen D H, and Chiu W (2012) Direct electron detection yields cryo-EM reconstructions at resolutions beyond 3/4 Nyquist frequency. J. Struct. Biol. 177, 589-601.

Briggs J A (2013) Structural biology in situ-the potential of subtomogram averaging. Curr. Opin. Struct. Biol. 23, 261-267.

Carroni M and Saibil H R (2016) Cryo electron microscopy to determine the structure of macromolecular complexes. Methods 95, 78-85.

Danev R and Baumeister W (2016) Cryo-EM single particle analysis with the volta phase plate. eLIFE 5, 13046.

Dubochet J, Adrian M, Chang J, Homo J, Lepault J, McDowall A W, and Schultz P (1988) Cryo-electron microscopy of vitrified specimens. Q. Rev. Biophys. 21, 129-228.

Frank J, Verschoor A, and Boublik M (1981) Computer averaging of electron micrographs of $40 \mathrm{~S}$ ribosomal subunits. Science $\mathbf{2 1 4}$, 1353-1355.
Grassucci R A, Taylor D, and Frank J (2008) Visualization of macromolecular complexes using cryo-electron microscopy with FEI Tecnai transmission electron microscopes. Nat. Protoc. 3, 330-339.

Li X, Mooney P, Zheng S, Booth C R, Braunfeld M B, Gubbens S, Agard D $A$, and Cheng Y (2013) Electron counting and beam-induced motion correction enable near-atomic-resolution single-particle cryo-EM. Nat. Methods 10, 584-590.

McMullan G, Faruqi A R, Clare D, and Henderson R (2014) Comparison of optimal performance at $300 \mathrm{keV}$ of three direct electron detectors for use in low dose electron microscopy. Ultramicroscopy 147, 156163.

Scheres S H (2012) RELION: implementation of a Bayesian approach to cryo-EM structure determination. J. Struct. Biol. 180, 519-530.

Schur F K, Hagen W J, Marco A, and Briggs J A (2013) Determination of protein structure at $8.5 \mathrm{~A}$ resolution using cryo-electron tomography and sub-tomogram averaging. J. Struct. Biol. 184, 394-400. 